\title{
Extending the team component of the Latimer ethical decision-making model for palliative care
}

This article was published in the following Dove Press journal:

Journal of Healthcare Leadership

3I March 20II

Number of times this article has been viewed

\author{
Mary Ellen Purkis' \\ Elizabeth Borycki ${ }^{1,2}$ \\ Craig Kuziemsky ${ }^{3}$ \\ Fraser Black ${ }^{4}$ \\ Denise Cloutier-Fisher ${ }^{5}$ \\ Lee Ann Fox ${ }^{6}$ \\ Patricia MacKenzie ${ }^{7}$ \\ Ann Syme ${ }^{1,8}$ \\ Coby Tschanz ${ }^{1,4}$
}

'School of Nursing, ${ }^{2}$ School of

Health Information Science,

University of Victoria, Victoria,

British Columbia; ${ }^{3}$ Telfer School of

Management, University of Ottawa,

Ottawa, Ontario; ${ }^{4}$ Victoria Hospice

Society, Victoria, British Columbia;

${ }^{5}$ Department of Geography, University of Victoria, Victoria, British Columbia;

${ }^{6}$ Kingston General Hospital, Kingston,

Ontario; ${ }^{7}$ School of Social Work,

University of Victoria, Victoria, British

Columbia; ${ }^{8}$ British Columbia Cancer

Agency, Vancouver Island Centre,

Victoria, British Columbia, Canada
Correspondence: ME Purkis School of Nursing, University of Victoria, Victoria, British Columbia, Canada

Tel: + I 25072 I 8050

Fax: + I 25072 I 7067

Email me purkis-hsddean@uvic.ca
Background: Each year more than 240,000 Canadians die from terminal and chronic illnesses. It is estimated that $62 \%$ of those deaths require palliative care. Palliative care is a specialized domain of health professional team practice that requires discipline-specific knowledge, skills, judgment, and expertise in order to address patient hopes, wishes, symptoms, and suffering. With the emergence of palliative care as a specialized area of interdisciplinary practice, new practice models have also emerged, eg, the Latimer ethical decision-making model for palliative care. The purpose of this research was to undertake a descriptive ethnographic field study of palliative care team practices to understand better the interdisciplinary team communication and the issues that arise when members of different health professions work together as a team.

Methods: Study data were collected by observing and videotaping palliative care team meetings. Data were then analyzed using direct content analysis.

Results: The study findings substantiated many of the team practice concepts outlined in Latimer's model. Palliative care teams engage in a number of processes that address patient symptoms, suffering, hopes, and plans. However, several new findings also emerged from the data that were not explicit in Latimer's original model. Teams employed five additional emergent team processes when addressing patient symptoms and suffering while attempting to fulfill patient hopes and plans. Those five team processes included explicating practice norms, leadership, provider assumptions, interdisciplinary teaching, and patient safety.

Conclusion: Although many team processes have been identified by practice models in the literature, there is a need to study the applicability of these models empirically to validate their representation of aspects of team practice.

Keywords: interprofessional team practice, palliative care, ethics, ethnography

\section{Introduction}

Palliative care is considered a specialized area of health professional practice. While many practitioners of health disciplines acknowledge its importance, as well as the need for and value of interprofessional teams in this practice context, explicit descriptions of such practice are difficult to find. Interprofessional teams are said to bring differing discipline-specific knowledge, skills, judgment, and expertise and provide a high quality of care aimed at addressing patient hopes, wishes, symptoms, and suffering. Because palliative care involves providing patients and their families with care during the chronic and terminal end stages of disease, there is a recognition that the primary role of palliative care is to enhance the management of the severity and intensity of symptoms (eg, symptom-modifying therapy for pain and reduced function). ${ }^{1}$ Consequently, health professional teams working in palliative care settings 
often deal with complex patient and family biopsychosocial and spiritual needs that require the expertise of many health care disciplines, ${ }^{2}$ and the need for health care professionals prepared to work effectively in such teams is growing as the population in our jurisdiction ages.

Canada's population is expected to age significantly over the next 10 years. Current estimates suggest that $16 \%$ of Canada's population will be over the age of 65 years by the year 2010. ${ }^{3}$ Indeed, Canada leads the developed world in projected increases in its elderly population by the year $2030 .{ }^{4}$ While there continues to be debate about the inevitability of increasing demands for health care with advanced aging, Davies and Higginson state that "if more people live to an older age, and if chronic diseases become more common with age, then the number of people in a population living with their effects will increase. This means that there will be more people needing some form of help towards the end of life". 5 Palliative care focuses on therapy that relieves suffering and/or improves the quality of life for patients with terminal illness. More than 240,000 Canadians die each year, ${ }^{3}$ and it is estimated that $62 \%$ of those deaths require access to hospice and palliative care services. ${ }^{6}$ The number of deaths in Canada is expected to increase by $33 \%$ by the year 2020 to more than 330,000 deaths per year. ${ }^{3}$ These estimates regarding the demand for palliative care services may be higher when one considers the demand for such care within the context of managing other chronic illnesses, such as chronic lung disease, chronic heart disease, and debilitating forms of osteoporosis, in addition to cancer. ${ }^{7}$

Historically in Canada, the provision of palliative care has been the responsibility of the patient's family. In the early 1970 s, formalized palliative care services involving health professional teams emerged in teaching hospitals in Winnipeg and Montreal in response to the complexities associated with palliative patient care management. Team meetings represent the primary organizational structure that supports teamwork in health care organizations. ${ }^{8}$ Team meetings provide members of each health discipline with the opportunity to meet to develop goals, objectives, plans, and solutions to complex patient care problems. ${ }^{9}$ From a health care organizational perspective, team meetings are "the basic prerequisites for collaboration", ${ }^{10}$ and communication and coordination of work. ${ }^{11}$

The success of the team approach in the palliative care setting as a health care delivery mechanism has led to its integration into a variety of health care environments including hospital, community, nursing home, long-term care, free-standing hospice centers, and home care. As a result, palliative care programs now provide patients and their families with a range of services, from pain and symptom management to bereavement and support services for families across Canada. ${ }^{12}$

The emergence of palliative care teams has led to research documenting the value of teamwork in the palliative care setting (eg, improved patient and family satisfaction with care and symptom control). Further to this, palliative care research involving the study of teams has found palliative care teams can reduce length of hospital stay and hospital costs associated with patient care. ${ }^{13}$ The use of palliative care teams has thus been advocated as an essential aspect of managing complex palliative care patients and families. Although the cost-effectiveness of team approaches has been demonstrated, the literature continues to describe the challenges associated with achieving positive patient care outcomes using interdisciplinary teams.

\section{Latimer ethical decision-making model}

With the emergence of palliative care as a specialized area of interdisciplinary team practice, new models of palliative care practice have also emerged, among them the Latimer ethical decision-making model for palliative care (Figure 1). Latimer's model ${ }^{14}$ suggests that seriously ill patients and their families receive care that "acknowledges the inherent worth and dignity of each person", and that care should be guided by the ethical principles of autonomy, beneficence, nonmaleficence, and justice, as outlined by Beauchamp and Childress. ${ }^{15}$ Therefore, patients are treated as unique individuals who have a right to compassionate care, gentle truth, decision-making autonomy, and physical and psychospiritual care. Latimer's model recognizes the dynamic nature of palliative care and the complex interplay between patient, family, disease process, and the health care system. Latimer suggests that health professionals need to engage in continual discussion and reflection with the patient and their family in order to provide high-quality palliative care. ${ }^{14,16}$

The Latimer model has been credited with preventing inadequate communication, physician withdrawal, patient

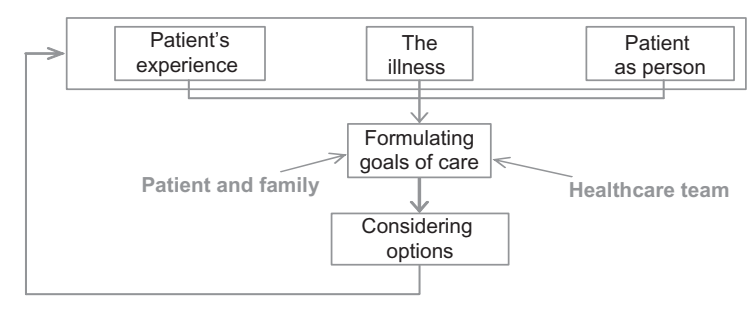

Figure I Latimer ethical decision-making model (9). 
labeling, and the provision of poor patient health care. ${ }^{14}$ Its emergence in the medical literature has led to its use and acceptance as a guideline for providing palliative care in Canada ${ }^{14,17,18}$ and the development of several palliative care management tools that aid in the care of terminally ill patients and their families. ${ }^{19,20}$ The model's success has also led to its adoption as a core underlying framework for the development of continuing medical education, ${ }^{19}$ an undergraduate course for educating students from the differing health care disciplines who practice palliative care, ${ }^{2}$ and is used in varying health care settings as a practice model by interdisciplinary palliative care teams (eg, hospice and hospital settings). As one of the only explicit models guiding the delivery of palliative care that addresses the context of health care delivery in Canada, the model was employed as a conceptual tool organizing a larger program of research of which this was one component. ${ }^{21}$

\section{Models representing palliative care teams}

The Latimer model has been successfully used by palliative care physicians and health professionals. However, little research has explored its use and relationship to interdisciplinary palliative care teamwork. Furthermore, although the Latimer model points to the interdisciplinary team as being the ideal avenue for formulating team goals and providing total patient care (including the physical, emotional, and spiritual), the model does not articulate the specific processes that an interdisciplinary health care team should undertake in providing patient care nor does it specify the disciplinary membership of the team. Similarly, other palliative care models do not specify team processes, eg, the "Square of Care" ${ }^{22}$ and Meghani's model. ${ }^{23}$ In summary, many palliative care team models do not specify team processes, membership, or outcomes associated with providing palliative care using interdisciplinary teams. Therefore, there is much to learn about palliative care team composition, processes, functions, and outcomes.

Health care delivery has in general been conducted in ways that encourage interdisciplinary teamwork. The recent focus on this form of practice may be signaling the need to review both the commitment to and accomplishment of interdisciplinary health care teams. ${ }^{24}$ Effective interdisciplinary team care delivery necessitates that teams coordinate their activities through communication. ${ }^{25}$ There is a need to understand whether such team communication processes might be organized and supported in particular ways to enhance care. There is also a need to identify the types of interventions made by team members and the optimal organizational design $(\mathrm{s})^{26}$ that would support effective team practice. De Haes and Teunissen ${ }^{1}$ point out there are few observational studies of team practice that focus on communication in palliative care and, to date, there are no studies that focus specifically on interdisciplinary communication processes. ${ }^{27}$

Despite the fact that there are numerous conceptual models about teams, there is little research that has looked at how these models translate into real clinical settings. The purpose of our research was to undertake a descriptive field study of team practices within the context of organizational team meetings undertaken in palliative care settings to understand better interdisciplinary team communication and the issues that arise when health care disciplines work together. The specific focus of this study was to explore interdisciplinary communication related to "transitions". We defined transitions as times during the course of providing care for the patient and family when decisions needed to be taken that have the effect of shifting the focus and/or direction of patient care. Morita et $\mathrm{al}^{28}$ identify this period as one experienced by most patients and their families as very distressing, and that their distress is largely underpinned by poor communication on the part of the care team.

\section{Methods}

The study reported here used field methods consisting of observation and video recording of team meetings ${ }^{29}$ to generate data for qualitative analysis. Team meetings were selected as the unit of analysis. Data collected during the team meetings were analyzed using directed content analysis. Directed content analysis involves first coding the data using a model or theory (see Figure 1). In cases where the model or theory does not adequately describe the data, additional qualitative codes are developed, defined, and used to code all subsequent observational and video data. ${ }^{30}$ More details about the data analysis are provided in the data analysis section of this paper.

\section{Data sources}

Access to two different palliative care teams was negotiated. Applications to the relevant human research ethics boards were submitted and approved. The two palliative care teams (A and B) were audiotaped and video recorded in two different institutions. Recording was done using a Sony ${ }^{\circledR}$ Mini DVD camera mounted on a tripod, as described by Kushniruk and Borycki. ${ }^{31}$ All recordings were transcribed verbatim. Team A was located in an inpatient hospice unit, while Team B worked in the outpatient department of an ambulatory cancer agency. Members of Team A met on a daily basis to discuss 
patient issues, but the focused team meeting where patients were reviewed in a systematic manner took place once each week and these are the meetings we observed. Similarly, members of Team B met frequently during each week to discuss pertinent patient issues as they arose, but met weekly for a systematic review of current patients, and this was the meeting we observed. Both teams met for approximately two hours, during which time we recorded their discussions.

Team A consisted of a physician, a physiotherapist, team leader (registered nurse), community case manager, counselor, spiritual care provider, and registered nurse. Team B consisted of three physicians (usually two palliative care specialists and a medical oncologist), a team leader (registered nurse), a counselor, and a pharmacist. The number of team members attending observed meetings varied from five to 10 people, and depended on the nature of the discussions (eg, patients who were discussed and involvement of differing interdisciplinary team members). In total, 10 team meetings (four from Team A and six from Team B) were observed and videotaped. Thirty patient cases in total were discussed during the meetings. We did not compare the work of the teams because the differences in the contexts of care (eg, inpatient hospice care versus ambulatory care) did not support such comparisons. Instead, the work of the teams in both settings was taken in total to represent a range of possible ways of accomplishing teambased palliative care.

\section{Data analysis}

A thematic analysis ${ }^{32}$ of transcribed team meetings was undertaken by three research team members (EMB, CK, MEP) who reviewed the transcripts individually at first, drawing out aspects of the team meetings that were reflected in the Latimer ethical decision-making model. ${ }^{14}$ Initially, all transcripts were analyzed using directed content analysis. Key concepts from Latimer's model, including "patient's experience", "the illness", "patient as person", "formulating goals of care", and "considering options", were used to code the transcripts.

Following this, the three research team members (EMB, CK, MEP) met and reviewed the transcripts coded by each researcher. This allowed the researchers to assess the consistency of the coding of the transcripts according to Latimer's model. The researchers then examined those parts of the transcript that could not be coded using concepts from Latimer's model. In cases where new concepts emerged from the data, the researchers discussed the new emergent concepts to obtain conceptual and definitional clarity. Once consensus was reached, the new concept was used in a recursive manner to code all transcripts.

In this analysis, we approached the transcripts as reflecting accounts of the everyday accomplishment of interdisciplinary palliative care team practice. ${ }^{33}$ We were particularly interested in determining how the focus of a patient intervention arose out of a team meeting. For example, the analysis involved being sensitive to both the role of those who could lead the team to a particular decision about care, as well as tracing the language used by that person to accomplish a particular outcome, including how other team members aligned with one another to secure, or undermine, particular outcomes. The research team spent a total of 50 hours reviewing, coding, and analyzing the data.

\section{Results}

The study findings substantiated many of the team practice concepts outlined in Latimer's model. It revealed palliative care teams engaged in a number of processes aimed at addressing patient symptoms, suffering, hopes, and plans, as outlined by Latimer, ${ }^{14,16}$ when formulating general and specific patient care goals. Several new findings also emerged from the data that were not explicit in Latimer's model, including five unique team processes. Those processes included explicating practice norms (including conveying organizational knowledge and negotiation), taking leadership (including focusing, transitioning, and building consensus), exposing provider assumptions, interdisciplinary learning, and patient safety. Figure 2 shows these processes and subprocesses within the context of the Latimer model. These five new or emergent processes with their associated subprocesses are expanded upon below.

\section{Process I: explicating practice norms}

Practice norms refer to ways of doing practice that are often not explicitly documented anywhere and have emerged through the experience of the interdisciplinary team process. Team members employed different strategies that resulted in alerting other team members that there was a "usual" way of proceeding with any sort of group meeting and that members of the team should respect those norms. For instance, we observed that one team had changed their usual format in order to accommodate the research team. Usually, all patient cases would be discussed but, in order to enable the team to focus on key cases and make better use of meeting time (to help the researchers out), it was decided (by a member or members of the team) that only 


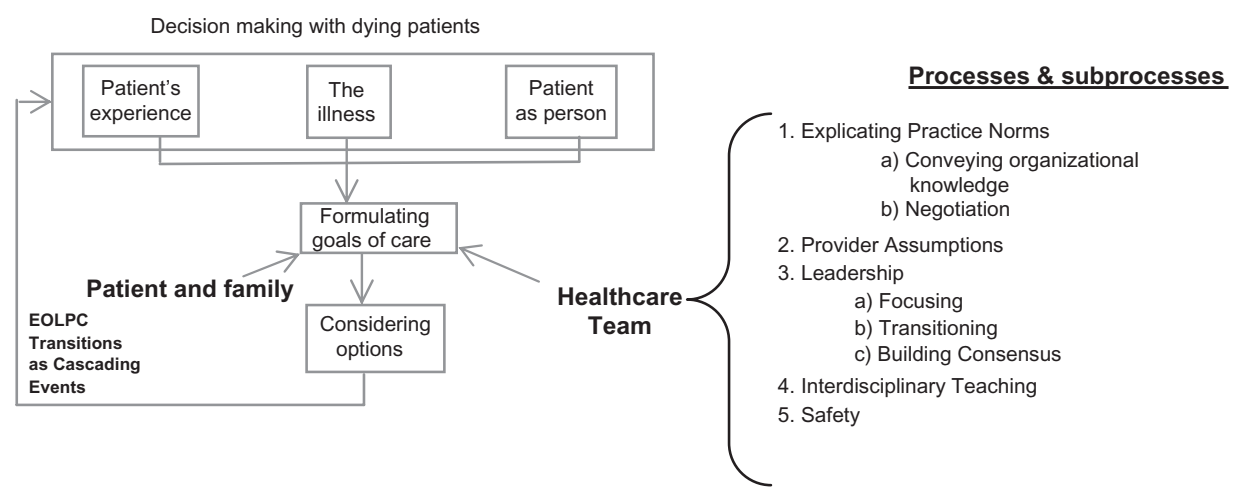

Figure 2 Team processes and sub-processes within the context of Latimer's model.

a few cases would be discussed when the researchers were present. As the example demonstrates, the teams engaged in additional work to accommodate the researchers. While in some research methods such accommodation may be understood as having a negative impact on the validity of the findings, from our ethnomethodological perspective, ${ }^{33}$ the accommodation reveals and exemplifies the particular feature of team practice where changes to the norm require a reorienting comment, usually from a team member who assumes leadership for the team process. In this instance, the revision to the usual process to accommodate the researchers was observed to have emerged through discussion as an "understanding" amongst team members. Even so, an exception to both these norms was also discerned, in that if one of the regular team physicians was away, the substitute physician is given more time to enable discussion of all of his/her cases. This observation underlines the ethnomethodological principle that actors engage in conversations that are structured through historical organizational practice and that change, whether planned or temporary to accommodate a research project, is extremely difficult to sustain. Note that all names used in the following scenarios are pseudonyms.

\section{Example I}

MD1: Is John coming?

RN1: No. Steve should be here soon. So we have a few [patients] we need to discuss today.

MD1: We sort of changed our format a bit in that we're not gonna talk about every patient, and we're just going to focus on the ones that are important, but for you, feel free to go though all yours

MD2: thanks .... .

Abbreviations: RN, registered nurse; $\mathrm{MD}$, physician.
A substitute physician would not have the same familiarity with the patient cases that the regular team physicians had. Therefore, to ensure that all patient needs were attended to, the interdisciplinary team allocated additional time to discussing patient cases with a substitute physician. In example 1, the regular team physician (MD1) makes that point clear by telling the substitute physician (MD2) to not feel rushed and feel free to go through all of his or her cases.

\section{Subprocess IA: conveying organizational knowledge}

In a similar way, we observed team members conveying organizational knowledge within team meetings. Conveying organizational knowledge refers to the act of communicating with team members about organizational policies, procedures, and processes for coordinating team activities. The researchers observed that, during team meetings, relevant information about differing organizational policies, procedures, and processes resided with differing disciplines and team members. Each would provide information he or she was aware of that influenced the collective choice of health care services aimed at providing optimal patient care. For example, one discussion involved the use of alternative medications and how patients are not always forthcoming about this.

\section{Example 2}

MD: $\quad$ I think people are saying ... that about 60 odd percent of people are on alternative medicine but they don't mention it, some people are now getting more used to the idea that it might conflict with some of the stuff they are getting from a doctor or an oncologist and therefore they are fessing up and saying "I'm taking this a little bit ..." 
TL: A lot of people assume herbal medicines are harmless because they're made of herbs ... but ...

Pharm: There's actually a database for free here because we have a subscription so anywhere on one of the computers, here you look on the library database, it's natural medicines comprehensive database and you can look up ... what's clinically known and it will also give you side effects and interactions ...

Abbreviations: MD, physician; TL, team leader (a registered nurse); Pharm, pharmacist.

In this instance, the pharmacist was aware of the subscription to the database of natural medicines but the rest of the team was not. By conveying that knowledge to the team and indicating that the database could be accessed from any computer by the team, the pharmacist provided the team with information that could promote better patient care.

\section{Subprocess IB: negotiation}

Perhaps because interdisciplinary practice norms are not written or explicit, practice norms are liable to be influenced by similarly unclear relationships of power. In cases, where team members disagreed or there was team member role overlap, team members would then engage in team-level negotiation that would facilitate decision-making. The following example illustrates such team-level negotiations. Here, the team is attempting to reach agreement by negotiating about the types of service (that would be provided by interdisciplinary team members to best meet patient needs):

\section{Example 3}

MD: Even though that happens at family meetings I wonder if a family meeting (is needed). If we could have one tomorrow or the next day and it may then become obvious to him that Friday or Thursday is too soon (to go home) but I wonder if that might be the only way he would realize that?

Couns: What time tomorrow would work for you?

MD: Uh. You're here until 1? Why don't we put them in. It's okay back to back.

Couns: To which back?

MD: How about 11:30? We'll keep Mr W's short and then go into the patient.

RN: If we can get the wife to come in.

MD: Yes, hopefully she will.

Abbreviations: RN, registered nurse; MD, physician; Couns, counselor.

In the above interchange, a physician and the counselor are trying to identify a time that is mutually agreeable to both parties to conduct a family meeting. The nurse indicates her support for a family meeting by stating she will try to get the wife to come for a meeting at that time.

In this example, the physician's interest in coordinating a family meeting is facilitated by other team members. The physician gives voice to a concern that had arisen during this team meeting that the patient is hoping to be able to return home, a wish not shared by the care team. The example demonstrates that the physician's interest in keeping the patient on the unit through the mechanism of a "family meeting" demands work of other team members, such as the nurse who will now have to coordinate the presence of the patient's wife in order to make this a family meeting.

\section{Process 2: exposing provider assumptions}

One challenge for palliative care teams is the complexity of patient cases. Palliative care teams also require a significant amount of data to make decisions where patient complexity is high. In our study, interdisciplinary teams used paper-based records. The paper records (in some cases) increased the length of time required to discuss a patient's case because of inaccuracies in the record and absence of some data. This led some providers to make assumptions (and errors) during interdisciplinary team discussions. The following example illustrates this occurrence well. In the example, a pharmacist has raised a question about a patient's medication by wondering if a drug should be discontinued because of another drug the patient was taking:

\section{Example 4}

RN1: Do you have any comments about any of today's patients that you want to ... ???

Pharm: Well, Mrs H-S, I guess, I'm questioning why she's on Tylenol 1, that can be discontinued if she's on oxycodone. SR oxycodone.

MD3: Is she still on it? I don't think she is, is she?

Pharm: She might be not be using it ... prn.

RN1: Yeah, she probably just has it in her cupboard now.

Pharm: And, (another pharm) wrote: "add gabapentin"?

MD1: Yeah, we were, we were going to do that but as I say, she's 83 years old so we were going along cautiously.

Abbreviations: MD, physician; RN, registered nurse; Pharm, pharmacist.

In the above example, both the pharmacist and physician have questioned whether the patient is taking Tylenol 1. However, that question is never actually 
answered because RN1 states "she probably just has it in her cupboard now" and then the team moves on to the next issue.

\section{Process 3: leadership}

The importance of effective team leadership cannot be overstated. In this study, team leadership was observed to have a crucial role in maintaining the focus of the team. Leadership, as a team process, accomplished two key team tasks during meetings, ie, leadership processes ensured meeting boundaries were maintained (eg, meetings were on time and content was covered) and leadership also ensured team tasks were articulated and someone was designated to be responsible for completing patient related tasks (this ensured team task clarity and facilitated task assignment to members of the team). Scenarios 1 and 2 below are illustrative of two occurrences where leadership was used to clarify and assign tasks to team members. The team leader in both examples was a nurse (ie, two different nurses). In both teams, nurses assumed the leadership role.

\section{Example 5, Scenario I}

RN5: So what's the procedure for getting him an oncologist in (Town Y)?

TL: Let the cancer clinic know

MD: The cancer clinic will normally ....

RN5: Is that Dr G? ... I just phone them and say this is what is happening and deal with it

TL: It's not our problem

MD: What you need to know is his address in (Town $\mathrm{X}$ )

RN5: Okay I'll do that ... and Dr $\mathrm{H}$, is it my responsibility to notify Dr H? He's also asked me to notify his GP

TL: $\quad$ Yes, just give him a call ... and tell him, that's all you need to notify, call and say it's [name] from Hospice

Abbreviations: RN, registered nurse; MD, physician; TL, team leader.

In scenario 1 described above, the team leader, after assigning the task of obtaining an oncologist to RN5, outlines the procedure for getting an oncologist. She provides RN5 with further guidance about the tasks she must undertake, including calling the cancer clinic and notifying other physicians involved in the patient's care regarding the referral to an oncologist. In assigning the task, the team leader also sets out the boundaries for the nurse in terms of contact between members of the team and an external group (eg, the cancer clinic) as well as other physicians (eg, the GP).

\section{Example 6, Scenario 2}

PT: $\quad$ So she doesn't need a home oxygen assessment then, is that what I'm hearing? Or we're not sure?

TL: Well you should demonstrate that ... her sats are falling then I think we would go to that place ... but there's no point in going there if your test shows that it's a non-issue

PT: $\quad$ Okay (writes down details)

RN2: (to PT) So [PT name] are you going to do some sats as she goes down the stairs or would you like me to do some as well?

PT: I'll do it and then I'll tell you how it looks

Abbreviations: RN, registered nurse; PT, physiotherapist; TL, team leader.

In scenario 2 outlined above, the team leader outlines the procedure for determining if an individual requires a home assessment for oxygen. In doing this, the team leader assigns a number of subtasks to the PT that are part of the task of demonstrating a need for oxygen. She makes the PT aware of the boundaries for team member work. In this case, she outlines when the boundary of the team extends to a patient's home (as there is a need for an oxygen assessment).

\section{Subprocess 3A: focusing}

The interdisciplinary palliative care teams in our study did make attempts to address immediate and future patient hopes, wishes, symptoms, and suffering. However, we observed that a good deal of discussion in team meetings made reference to organizational processes or activities taking place outside of the team's sphere of influence that were affecting a patient's hopes, wishes, symptoms, and suffering. In those instances, a team member or the team leader would focus the discussion back to addressing the patients' symptoms, suffering, hopes, and wishes within the current organizational context. Here, a team member encouraged the team to move their attention to specific patient's symptoms (ie, focusing the discussion on the patient's symptoms) that were originally being discussed before the topic or discussion shifted away to organizational processes that might influence patient care. This was done in order to obtain physician orders for medications that could help alleviate the patient's symptoms:

\section{Example 7}

RN: I noticed she is a little less settled ... that UTI went up quickly.

TL1: Are you saying you need more orders?

Abbreviations: RN, registered nurse; TL, team leader. 
In this short interchange we see the registered nurse drawing attention to the sudden emergence of a urinary tract infection. While this is a relevant observation for this health care professional to make, the team leader works to bring focus to the observation. The most relevant thing for the patient is not the speed of an infection becoming evident, but tracking down the team member, in this instance the physician, who can write an order for antibiotics to address the infection as quickly as possible.

\section{Subprocess 3B: transitioning}

There were many opportunities for wide-ranging discussions about patients during interdisciplinary team meetings. During interdisciplinary team discussions, team leaders and team members needed to determine when a sufficient amount of patient-related discussion had occurred about each patient before moving on to the next patient. As Gersick ${ }^{34}$ notes, a "group" progress was triggered more by members' awareness of time and deadlines than by completion of an absolute amount of work in a specific developmental stage". We have found similar processes at work when a team member plays the role of time-keeper and, when sufficient time has been spent on a case, a transitioning comment may effectively and efficiently help the team to change gears and move on:

\section{Example 8}

MD: The son of the patient that just died was apparently quite upset at [female patient] for involving herself in their conversation ... you know she would chip in and he got quite angry and said 'if you don't tell her to shut up I will', thinks it was a bit rude

Couns: But he had challenges ...

MD: Oh sure he did ... .

TL: Okay that's it, room 341 I guess that's you (to RN2)

RN2: Yes we have [female patient] she is a 77 year old who came to us for pain control that she couldn't manage at home ...

Abbreviations: RN, registered nurse; MD, physician; Couns, counselor; TL, team leader.

In this instance, it is the team leader who effects the transition, moving the conversation from a discussion involving one patient and her family to another. She ends the discussion of one case by stating "okay that's it" and, naming a room number ("room 341") and addressing one of the team members ("I guess that's you [to RN2]"). The team leader effectively transitions to another case. Furthermore, as Gersick notes, the nurse leader may have based this transition on an awareness of time and deadlines rather than on the completion of any particular group activity. Transitioning was also used as a method of moving away from any challenges encountered by the team.

\section{Subprocess 3C: building consensus}

Although team members have discipline-specific knowledge and expertise, there is often some overlap with other team members. In our study, if team member opinions differed, there was an attempt to obtain consensus by identifying an approach that would best meet the patient's needs.

\section{Example 9}

Pharm: Well, Mrs. H-S, I guess, I'm questioning why she's on Tylenol 1, that can be discontinued if she's on oxycodone. SR oxycodone.

MD3: Is she still on it? I don't think she is, is she?

Pharm: She might be not be using it ... prn.

RN1: Yeah, she probably just has it in her cupboard now. Pharm: And, another pharm wrote: "add gabapentin"?

MD1: Yeah, we were, we were going to do that but as I say, she's 83 years old so we were going along cautiously.

Abbreviations: MD, physician; RN, registered nurse; Pharm, pharmacist.

Although we have used this example earlier to illustrate team members' practices of exposing provider assumptions, it is useful again here to show how consensus is accomplished. It may be helpful here to make a side methodological reference to the fact that when researchers collect large amounts of "naturally occurring" conversation between professionals within a highly focused field such as palliative care, it is often the case that these research materials reveal more than just one theme. ${ }^{35}$

In this example, the pharmacist questions why the patient is receiving Tylenol 1 in addition to another medication (sustained-release oxycodone). The pharmacist suggests another medication (eg, gabapentin). It may be that the "error" that has been exposed (ie, continuing a prescription for Tylenol 1 that is no longer necessary, given subsequent prescribing actions) needs to be resolved and consensus reached that the plan of care is now agreed to by everyone on the team. In this example, we note that the physician plays this consensus-building role by stating that the team discussed the idea of making further prescriptive changes previously but wanted to be cautious in adding the additional medication because of the patient's age. 


\section{Process 4: interdisciplinary teaching}

Another characteristic of interdisciplinary team practice is team teaching. On several occasions, one or more team members would attempt to explain or clarify information presented at the team meeting thereby "teaching" other members of the team.

\section{Example 10}

Couns: ... but she said she was hungry for the first time, she said she ate tons [Team laughs. They appear happy about what the patient ate]

RN: It's kind of like true confessions, "I ate this and I drank this"

MD: $\quad$ She just had radiation to her liver for her liver mets ... her liver was damaged by metastases and then further damaged by radiation ... her serum albumin is 14 which is incredibly low and I think that's the basis for her leg edema so it probably wouldn't respond all that well to a diuretic, as a diuretic would drive her sodium down further so we're in a bit of a tight spot with regard to her feet .... .

PT: $\quad$ And in terms of ... she's a fall risk and I discussed that with her just because of her electrolytes and blood pressure and what not ... and she's been telling me she's been calling [via call bell] and she's reliable with that ...

Abbreviations: MD, physician; RN, registered nurse; PT, physiotherapist; Couns, counselor.

Initially, the physician teaches the team about the impact of the radiation and cancer on the patient's liver and how it led to leg edema. The physician goes further to teach the team about why the patient would not respond to a diuretic and how administering a diuretic might negatively affect the patient's health (eg, drive her sodium down further).

In the next example, a physician team member attempts to teach his colleagues about radiation therapy.

\section{Example II}

MD: ... his wife was concerned about him falling, using a walker, it was interesting that she said that every time he had some surgery he seemed to have lost a few brain cells, yeah I think in the past ... I've seen people with prolonged surgery who don't seem to be quite as bright after the surgery, whether it was the anesthetic or they were hypotensive for a period of time or whatever ...
ROMD: We're seeing it more and more with chemo but we just didn't recognize it before either, they are toxic drugs that do affect the brain, "chemo brain" is way more common than we used to imagine, and now that we ask about it we see depression, emotional lability, memory loss, people that can't do crosswords anymore (...)

MD: I think people are living longer and some of these things take time to manifest ... it's like brain radiation sometimes people become quite demented eventually if they live long enough

ROMD: This, this "chemo brain" is acute, it comes on the first course ... I've had patients that have been on 5 -FU for a week and they started noticing memory loss, emotional changes ...

Abbreviations: MD, physician; ROMD, medical oncologist.

Here, the radiation oncologist shares his or her knowledge about the effects of chemotherapeutic regimes on patients. The other physician initially listens to his colleague and adds that these negative effects arise over time, and perhaps can be attributed to the fact that treatments result in people living longer. The radiation oncologist pushes this discussion further by claiming that the cognitive impacts are observed to occur with the very first course of chemotherapy. Such discussions could open up opportunities for team members to learn about the relative value and impact of treatments over time.

\section{Process 5: safety}

Safety concerns for drug effects are common topics of conversation within team meetings as seen in the following example:

\section{Example 12}

Pharm: She's still on valproic acid.

MD: Are you sure?

Pharm: [nods] Still on valproic acid, we started at $250 \mathrm{mg}$ three times a day for her ... pain ...

MD: Jaw pain

Pharm: Uhhm and if we're going to continue that I wonder if we should start doing liver enzymes? Cause that's supposed to be monitored ... liver and valproate

MD: What are we going to do about it if she has raised liver enzymes?

Pharm: Taper her off, try something else?

MD: [shrugs with small nod] Didn't we try something else with her previously?

Pharm: [consults paper medicine log] She had ... .

MD: Was she on gabapentin for a while? 
Pharm: No, at one point I thought we were doing liquid because her mouth was so small ... that was a long time ago so Gabapentin wasn't ... I don't think that was in the picture at all because of the swallowing

MD: She had difficulty swallowing?

Pharm: Back then, and it was really sensitive

MD: Well we can do liver enzymes and see ...

Abbreviations: MD, physician; Pharm, pharmacist.

In the above example, the physician decides that, despite the potentially damaging effects of the medication to the patient's liver, its positive effects outweigh the risks. However, the pharmacist team member challenges the decision feeling that no matter what stage the patient is at in the disease process, the monitoring of liver function should still be undertaken because alternative drugs may be prescribed. The physician maintains the role of primary provider by agreeing, perhaps somewhat reluctantly, to have the tests done to determine the impact of the current prescription on the patient's liver function. He or she concludes by leaving any change in prescription as something that might be determined at a later time.

\section{Discussion}

McCallin ${ }^{36}$ notes that "... research explaining how interdisciplinary team members manage their concerns and work together in everyday practice is minimal". We present our findings then as an empirical contribution to this research base on how team members manage their work together within a particular sociopolitical context, ie, that of one palliative care team working in an inpatient hospice setting and one in an ambulatory pain and symptom management clinic, both located in an urban setting in Western Canada. Taking McCallin's view that what is applicable in one "country" may not be transferable, the findings from this pilot study offer only the beginning of an understanding of how interdisciplinary teams function. In our description of five processes of teamwork, we have sought to provide an extension of Latimer's ${ }^{14}$ model of team-based palliative care, which has important implications for both practice and policy.

\section{Implications for practice}

First, these five processes of team practice afford a relatively unique glimpse into the complex and problematic terrain of interdisciplinary teams. As our study demonstrates, the capacity of team-based forms of practice to produce positive outcomes for patients and families is not immediately apparent. We observed practices that did coincide with emerging criteria for interdisciplinary practice, such as maintaining a focus on the person who is the recipient of care rather than on long-standing interdisciplinary rivalries, and on ensuring that teams have effective leadership. At the same time, we observed many instances where members of teams consistently remained silent during team meetings, apparently unable to bring the voice of their professional perspective to the table to contribute to the discussion. We also observed numerous occasions where, as we noted in the section on Teaching, the implications of differences between professional perspectives was not taken further than the stating of the difference. The implications of differences in perspectives in relation to the quality of patient care did not occur in any consistent manner. Thus, while there are clear political interests in advancing interdisciplinary care as a preferred form of practice to improve population health, improve access to care, and improve the efficiency and effective employment of health human resources, ${ }^{37}$ our field study demonstrates that these outcomes will not automatically occur simply because teams exist. Additional effort appears to be necessary in order to obtain the proposed advantages of team practice.

The second reason why the description offered here is important is because we have provided empirical insight into the processes of interdisciplinary teams. Although numerous conceptual models on teams exist, such as the Latimer model, to date there is little research that looks at those models in the context of real care delivery. A key message is that conceptual models must be viewed in the specific contexts where health care delivery takes place. The five additional processes of interdisciplinary team practice identified in this paper offer empirical insight to enhance the study of team practices and team practice models in different settings.

\section{Implications for policy}

The findings from this paper have implications for the design of policy and information and communication technologies to support interdisciplinary teams. A policy implication is the extent that teaching takes place within teams. Teaching was seen to be a valuable process in team-based care, but the extent of teaching is influenced by human resource issues. For example, in example 11, a medical oncologist provides a description of "chemo brain" in a teaching moment. There were other teaching moments provided by the medical oncologist, and despite the fact the patients being discussed were not his patients directly, he was still a valuable contributor to the team meetings. However, the medical oncologist was not able to attend all team meetings because the team meetings conflicted with his patient schedule. Furthermore, the team 
meetings were not part of his fee schedule and thus he could not bill to attend the meetings. Other studies have similarly pointed to funding issues as barriers to teamwork. ${ }^{38}$ Dedicated funding and fee payments for interdisciplinary team meetings would make it easier for team members to attend meetings and would support tasks such as team teaching.

\section{Directions for future research}

A common issue experienced by both teams we studied was poor access to data. In complex interdisciplinary care, such as palliative care, the access and sharing of data can be problematic because there are often multiple care providers and settings. The patient's medical records, which are largely paper-based, may be transferred across different settings and updated frequently within those different settings. As shown in processes 2 and 5, mediation safety was as issue because teams sometimes did not have access to requisite data to ensure safe care delivery. In other instances, there was information available but that was not known to team members, an example being the pharmaceutical database described in subprocess $1 \mathrm{~A}$.

In contemplating the next phase in this research process, our developing argument is that training modules designed to enhance high-quality team functioning in palliative care need to include opportunities for interdisciplinary teams to reflect upon their current practices in relation to these themes and processes. If team practice has not developed explicit strategies in relation to these themes, then strategies should be developed to enhance team functioning and, as a result, the quality of patient outcomes.

A shortcoming of this paper is that it was based on palliative care teams in two settings. Additional team processes, subprocesses, and contextual considerations may emerge in other settings. Future research will involve developing explicit practice strategies for interdisciplinary teams that will extend the model of palliative care espoused by Latimer. ${ }^{14}$ Such strategies will help us move towards common approaches for the design and evaluation of interdisciplinary teams.

\section{Conclusion}

The descriptions of interdisciplinary practice presented here mark an important step in the process of developing and extending models for interdisciplinary team practice that rely not only on conceptual frames that offer moral direction for practice, but also on empirical support for practitioners interested in improving the care they deliver to their patients in periods of transition during illness.

\section{Disclosure}

The authors report no conflicts of interest in this work.

\section{References}

1. De Haes H, Teunissen S. Communication in palliative care: A review of recent literature. Curr Opin Oncol. 2005;17:345-350.

2. Latimer E, Deakin A, Ingram C, O'Brien L, Smoke M, Wishart L. An interdisciplinary approach to a day-long palliative care course for undergraduate students. CMAJ. 1999;161:729-731.

3. Population and demography. In: Statistics Canada. Ottawa: Government of Canada; 2007.

4. Kinsella K, Velkoff V. An aging world: 2001. In: US Census Bureau. Washington, DC: US Government Printing Office; 2001.

5. Davies E, Higginson IJ, editors. Better Palliative Care for Older People. Copenhagen: World Health Organization; 2004.

6. Fact sheet: Hospice Palliative Care in Canada. Ottawa: Canadian Hospice and Palliative Care Association; 2007.

7. Sepulveda C, Marlin A, Yoshida T, Ullrich A. Palliative care: The World Health Organization's global perspective. J Pain Symptom Manage. 2002;24(2):91-96

8. West MA, Poulton BC. A failure of function: Teamwork in primary health care. J Interprof Care. 1997;11:205-216.

9. Patel VL, Cytryn KN, Shortliffe EH, Safran C. The collaborative health care team: The role of individual and group expertise. Teach Learn Med. 2000;12(3):117-132.

10. Drach-Zahavy A, Somech A. Understanding team innovation: The role of team processes and structures. Group Dyn. 2001;5:111-123.

11. Shortell SM, Kaluzny AD. Health Care Management: Organization Design and Behaviour. Scarborough, ON: Delmar/Thomson Learning; 2006.

12. Progress report on cancer control in Canada. In: Health Canada. Ottawa: Government of Canada; 2005.

13. Hearn J, Higginson IJ. Do specialist palliative care teams improve outcomes for cancer patients? A systematic literature review. Palliat Med. 1998;12:317-332.

14. Latimer E. Caring for seriously ill and dying patients: The philosophy and ethics. CMAJ. 1991;144:859-867.

15. Beauchamp TL, Childress JF. Principles of Biomedical Ethics. 3rd ed. New York, NY: Oxford University Press; 1989.

16. Latimer EJ. Ethical care at the end of life. CMAJ. 1998;158:1741-1747.

17. Downing M, Wainwright W. Medical Care of the Dying Patient. 4th ed. Victoria, BC: Victoria Hospice Society; 2006.

18. MacDonald N. Ontario palliative care statement: A template for the rest of Canada. CMAJ. 1993;148:891-893.

19. Latimer E, McDonald J, Krauser J. Toward the provision of effective palliative care in Ontario. In: OMA Colloquium on Care of the Dying Patient. Toronto: Canadian Medical Association; 1996.

20. Latimer EJ, Crabb MR, Roberts JG, Ewen M, Roberts J. The patient care traveling record $(\mathrm{R})$ in palliative care: Effectiveness and efficiency. J Pain Symptom Manage. 1998;16(1):41-51.

21. Lau F, Downing GM, Lesperance M, Shaw J, Kuziemsky C. Use of palliative performance scale in end-of-life prognostication. J Palliat Med. 2006;9(5):1066-1075.

22. Ferris FD, Balfour HM, Bowen K, et al. A model to guide patient and family care: Based on nationally accepted principles and norms of practice. J Pain Symptom Manage. 2002;24(2):106-123.

23. Meghani SH. A concept analysis of palliative care in the United States. J Adv Nurs. 2004;46(2):152-161.

24. Herbert C. Changing the culture: Interprofessional education for collaborative patient-centred practice in Canada. J Interprof Care. 2005; 19(S1): $1-4$.

25. Toussaint PJ, Coiera E. Supporting communication in health care. Int J Med Inform. 2005;74(10):779-781.

26. Jack B, Hillier V, Williams A, Oldham J. Hospital based palliative care teams improve the insight of cancer patients into their disease. Palliat Med. 2004;18(1):46-52. 
27. Marsella A. Exploring the literature surrounding the transition into palliative care: A scoping review. Int J Palliat Nurs. 2009;15(4): 186-189.

28. Morita T, Akechi T, Ikenaga M, et al. Communication about the ending of anticancer treatment and transition to palliative care. Ann Oncol. 2004;15(10):1551-1557.

29. Merriam SB. Qualitative Research: A Guide to Design and Implementation. San Francisco, CA: Jossey-Bass; 2009.

30. Hsieh HF, Shannon SE. Three approaches to qualitiative content analysis. Qual Health Res. 2005;15(9):1277-1288.

31. Kushniruk A, Borycki EM. Low-cost rapid usability engineering: Designing and customizing usable health information systems. Healthcare Quarterly/Electronic Healthcare. 2006;5(2):98-102.
32. Aronson J. A pragmatic view of thematic analysis. Qual Rep. 1994;2(1).

33. Garfinkel H. Studies in Ethnomethodology. Englewood Cliffs, NJ: Prentice Hall; 1967.

34. Gersick C. Time and transition in work teams: Toward a new model of group development. Acad Manage J. 1988;31(1):9-41.

35. Daft RL, Wiginton JC. Language and organization. Acad Manage Rev. 1979;4(2):179-191.

36. McCallin A. Interdisciplinary practice - a matter of teamwork: An integrated literature review. J Clin Nurs. 2001;10(4):419-428.

37. Health care system. In: Health Canada. Ottawa: Government of Canada; 2007.

38. Mulvale G, Bourgeault IL. Contextual factors affect collaborative mental health care in Ontario. Can Public Policy. 2007;33:s49-s63.

\section{Publish your work in this journal}

The Journal of Healthcare Leadership is an international, peer-reviewed, open access journal focusing on leadership for the health profession. The journal is committed to the rapid publication of research focusing on but not limited to: Healthcare policy and law; Theoretical and practical aspects healthcare delivery; Interactions between healthcare and society and evidence-based practices;
Interdisciplinary decision-making; Philosophical and ethical issues; Hazard management; Research and opinion for health leadership; Leadership assessment. The manuscript management system is completely online and includes a very quick and fair peer-review system. Visit http://www.dovepress.com/ testimonials.php to read real quotes from published authors.

Submit your manuscript here: http://www.dovepress.com/journal-of-healthcare-leadership-journal 